\title{
Clinical utility of endurance measures for evaluation of treatment in patients with mucopolysaccharidosis VI (Maroteaux-Lamy syndrome)
}

\author{
Arline McDonald ${ }^{\mathrm{a}, *}$, Robert Steiner ${ }^{\mathrm{b}}$, Kerry Kuehl ${ }^{\mathrm{c}}$ and Sean Turbeville ${ }^{\mathrm{d}}$ \\ ${ }^{a}$ Department of Preventive Medicine, Northwestern University Feinberg School of Medicine, IL, USA \\ ${ }^{\mathrm{b}}$ Department of Pediatrics, Oregon Health and Science University, OR, USA \\ ${ }^{\mathrm{c}}$ Human Performance Laboratory, Oregon Health and Science University, OR, USA \\ ${ }^{\mathrm{d}}$ BioMarin Pharmaceutical Inc, CA, USA
}

Accepted 3 March 2010

\begin{abstract}
Increased functional capacity of major organ systems improves the quality of life and contributes to reductions in the morbidity associated with chronic debilitating diseases. Routine endurance tests can be used to gauge the progression of disease and the impact of therapeutic modalities in disorders with multiple organ system involvement such as with Mucopolysaccharidosis type VI (MPS VI). MPS VI is a progressive disorder affecting multiple organs and tissues due to the deficient activity of $N$-acetylgalactosamine-4-sulfatase leading to the accumulation of glycosaminoglycan (GAG) dermatan sulfate. Since 2005, enzyme replacement therapy (ERT) with human recombinant $N$-acetylgalactosamine-4-sulfatase (galsulfase) has been an available treatment option for MPS VI. These patients are routinely evaluated for extent of disability, disease progression and the impact of ERT. Evaluations are made by a combination of urinary GAG measurement and submaximal intensity endurance tests such as the 3-minute stair climb (3-MSC), and the 6- and 12-minute walk tests (6-MWT and 12-MWT). This review highlights the clinical validity of endurance measures as inexpensive diagnostic tools for diseases affecting multiple organ systems and evaluating the impact of therapeutic modalities, such as ERT for MPS VI.
\end{abstract}

Keywords: Mucopolysaccharidosis, Maroteaux-Lamy Syndrome, enzyme replacement therapy, glycosaminoglycan, endurance tests, quality of life

\section{Introduction}

Improvement in the functional status of major organ systems is a primary therapeutic goal in the management of chronic debilitating diseases. Increased functional capacity improves the quality of life and con-

\footnotetext{
*Address for correspondence: Arline McDonald, Adjunct Assistant Professor, Department of Preventive Medicine, Northwestern University Feinberg School of Medicine, 680 North LakeShore Drive, Chicago, IL 60611, USA. Tel./Fax: +1 312304 1261; E-mail: a-mcdonald@northwestern.edu.
}

tributes to reductions in the morbidity and mortality associated with these diseases [1,38]. Endurance can be considered a surrogate marker of functional capacity because it is a quantifiable measure of the available reserves of the major organ systems that support activity [54]. Measures of endurance obtained from performance testing at submaximal intensity levels have been used clinically to assess functional status of the cardiovascular, pulmonary, and musculoskeletal systems, as well as to detect alterations in the operation of certain components of the nervous system [32]. These measures have been validated against standard clinical as- 
sessment endpoints and found to be strong prognostic indicators of responses to treatment, and of morbidity and mortality in patients with moderate to severe heart and lung diseases $[4,9,13,25,38,39,47,49,54,56$, $61,64,70,76]$. Performance tests have also been used to assess clinical status of adult patients with conditions as diverse as stroke $[34,35,57,74]$, chronic fatigue syndrome [53], fibromyalgia [58,65], multiple sclerosis [33] and spinal disorders [40,55,69], and of children with scoliosis [55,69] and juvenile idiopathic arthritis $[41,48]$.

As a measure of the integrative activity of multiple organ systems, endurance can be used to gauge functional capacity in disorders with multiple organ system involvement. Mucopolysaccharidosis type VI (MPS VI; Maroteaux-Lamy Syndrome) is a progressive disorder affecting multiple organs and tissues that is caused by a deficiency of $N$-acetylgalactosamine-4-sulfatase, a lysosomal enzyme responsible for the degradation of the glycosaminoglycan (GAG) dermatan sulfate [52]. In the absence of this enzyme, the stepwise degradation of dermatan sulfate is blocked resulting in intracellular accumulation of partially degraded substrate in the lysosomes of specific tissues. Cardiac abnormalities, particularly involving the mitral and aortic valves, are frequently observed and can be severe in patients with MPS VI [21]. Thickening of the mitral valve with regurgitation or stenosis is the most common cardiac lesion in this disease.

Upper airway obstruction, clouded corneas, and nerve compression syndromes are also found. Skeletal abnormalities appear to be the most intractable component of MPS VI [30]. Age of MPS VI diagnosis is variable depending on disease progression. Rapidly advancing disease is generally diagnosed within the first year of life whereas those with slowly advancing disease can be diagnosed at a later age [20]. Cardiorespiratory failure secondary to cardiovascular involvement and upper airway obstruction is the most common cause of death.

\section{Physiology of endurance}

Endurance is a measure of the level of effort required to perform an activity and therefore of the functional reserves of the cardiovascular, pulmonary or musculoskeletal systems $[41,48]$. Since endurance represents a coordinated activity of multiple organ systems, it captures the additive effects of functional deficits in more than one system. Patients with compromised car- diopulmonary and musculoskeletal function require a considerably greater effort to engage in routine activity than healthy individuals because they must utilize a larger proportion of their maximal capacity to perform these tasks [15,54]. Changes in endurance are therefore good indicators of changes in the functional reserves of these organ systems and support the therapeutic goal of improving endurance in the management of diseases with cardiovascular, pulmonary or musculoskeletal involvement.

Endurance testing is especially sensitive to cardiovascular and pulmonary function because the efficiency of aerobic energy metabolism, the limiting factor for endurance, is dependent on tissue perfusion and oxygenation $[42,46,48]$. Aerobic pathways draw primarily on the relatively unlimited reserves of fatty acids as fuel sources and thus can sustain low to moderate activity for longer periods compared with anaerobic metabolism which relies more heavily on the limited reserves of glycogen and is thus better suited for supporting short-term, high intensity activities. To sustain aerobic activity, cardiopulmonary function must be able to support the increased circulatory and ventilatory demands to insure both a steady supply of oxygen to fuel working muscles and an efficient removal of the carbon dioxide waste they produce [41].

Functional deficits in cardiovascular performance during activity prevent the incremental increase in cardiac output needed for a given level of effort resulting in insufficient tissue perfusion and oxygenation and early onset fatigue $[25,42,76]$. Compromised pulmonary ventilatory capacity reduces the efficiency of gaseous exchange between alveolar and ambient air causing accumulation of plasma carbon dioxide and the subsequent fall in plasma $\mathrm{pH}$ which inhibits aerobic metabolism. As a compensatory response, respiration rate is increased to facilitate removal of carbon dioxide and prevent a further decline in $\mathrm{pH}$ [41]. Patients with compromised ventilatory capacity frequently develop dyspnea during mild exertion limiting the type and duration of activities they are able to perform.

Abnormalities in musculoskeletal function impact endurance by compromising the effectiveness of cardiopulmonary responses to activity, impairing motor skills, and reducing the amount of aerobic energy available during activity. To function efficiently, the cardiopulmonary system depends upon the peripheral musculature to facilitate venous return to ease the workload on the heart and lungs. Muscle and joint function determine the degree of mobility and range of motion, and therefore limit the type and extent of 
activities that may be performed. Poor dexterity due to skeletal abnormalities increases the level of difficulty and amount of effort required to perform basic tasks. Since working muscles generate the major proportion of aerobic energy utilized during activity, skeletal abnormalities that limit mobility will reduce the supply of aerobic energy produced during activity and contribute further to the difficulty of performing routine tasks [14, 42]. Muscle atrophy, abnormal metabolic responses, and reduced enzyme levels are as important to determining the time to onset of fatigue during activity as underperformance of the cardiopulmonary system, and diminished endurance capacity caused by impairments in motor function cannot be completely offset by improvements in cardiopulmonary function $[38,75,76]$.

\section{Endurance testing in clinical settings}

Endurance tests are designed to measure outcomes that reflect the efficiency of performing an activity. Performance is influenced by the amount of effort expended which can be estimated from the heart rate achieved during the activity. Submaximal intensity tests require a smaller increase in heart rate than maximal intensity tests and thus are more appropriate for patients with functional impairments due to disease [7,17,22, $31,32]$. In clinical settings, endurance tests are usually performed at submaximal intensity levels because they are more representative of the amount of effort required to engage in routine activities. Submaximal intensity tests are safer to perform, have fewer contraindications, and can better accommodate physical limitations caused by pain, abnormal gait, or poor balance $[19,40$, $45,63,69,77]$. These tests are also more sensitive indicators of endurance for patients with diminished cardiopulmonary reserves who are utilizing close to their maximal capacity to perform basic tasks [15], and for special populations such as children who have congenital defects or disabilities that restrict activity $[19,40$, 69,77].

Walk tests are the most widely used of the submaximal intensity tests for assessment of endurance in clinical settings. These tests measure distance walked over a fixed time interval (e.g., 6 or 12 minutes) expressed as an absolute value or as a percentage of baseline values. The 12-minute walk test (12-MWT) was the first of these tests to be used clinically more than 30 years ago to measure endurance in patients with chronic bronchitis $[43,44]$. This test was adapted from the 12-minute treadmill test originally developed by Cooper [11] to measure fitness in healthy young men. The 12-MWT is a simple and highly reproducible test for measuring endurance in clinical settings and can be administered to patients with chronic diseases at all levels of severity $[6,43,44]$. The $12-$ MWT is more broadly applied than walk tests of shorter durations such as the 2-minute walk test (2-MWT) or 6-minute walk test (6-MWT) because of its wider dynamic range which allows changes in speed to be measured in patients able to walk 12 minutes at baseline and changes in distance to be measured in those who cannot [28]. A comparison of results obtained from walk tests of different durations found increased random variation when measurements were made over longer time intervals suggesting that the 12-MWT was more discriminating than the 2-MWT or 6-MWT [6].

Walk tests are adaptable for use in clinical settings because they are simple to administer and do not require expensive or complicated equipment or specialized training. The primary requirement for the test is a premeasured, level, and unobstructed space for walking. To perform the test, patients are instructed to cover as much ground as they can before they are unable to continue [17,24]. Because patients are allowed to set their own pace, rest as needed, and use any mobility device they normally use, walk tests are more representative of functional capacity utilized in performing routine daily tasks than other types of endurance tests. Before the test is administered, patients are familiarized with the walking path and any ancillary measurements that may be made during the test such as pulse oximetry or rate perceived exertion using the Borg scale [67].

The validity and reliability of walk tests can be influenced by a learning or training effect or by motivational factors such as encouragement or fear [35]. Familiarity with the walk test can result in improvements in scores which may be unrelated to physiological changes in endurance. Walk scores may also be improved by encouragement which is a powerful motivator to do better particularly among children. Fear about later pain and discomfort is a negative motivator which may prevent patients from exerting themselves fully during the tests. These limitations can be overcome by use of practice tests, standardization of encouragement practices, and techniques that directly address fears about the tests. Practice tests can minimize the impact of training on walk scores while standardization of timing and content of encouragement can minimize variability in these scores.

The 3-minute stair climb (3-MSC) test is an alternative measure of endurance that also accommodates 
multiple impairments in cardiopulmonary and musculoskeletal function and severely restricted mobility. The 3-MSC was adapted for children with MPS VI from the 3-minute step test, a validated measure of respiratory capacity in children with cystic fibrosis, taking into account the often severe physical limitations and special safety needs of children with MPS VI [2,28]. Although not a standardized test, the 3-MSC has been used historically as part of preoperative assessment to gauge lung function in patients undergoing lung resection [28]. The 3-MSC requires patients to climb as many stairs as possible over a 3-minute period allowing use of handrails and rest periods as needed. A statistically significant linear correlation has been demonstrated between the 3-MSC and the 12-MWT in children with MPS VI.

\section{Clinical validation of endurance tests}

The clinical validity of using endurance tests to assess functional status in different patient populations is supported by data showing statistically significant correlations between the results of these tests and validated physiological measures of functional capacity and standard clinical endpoints. Maximal oxygen uptake $\left(\mathrm{VO}_{2} \mathrm{max}\right)$ is considered the best objective predictor of the functional limitations of the cardiopulmonary system because the volume of oxygen consumed during maximal intensity exercise is dependent upon the mechanical efficiency of the heart and lungs $[13,23,46]$. Walk distances measured by the 6-MWT have been significantly correlated with $\mathrm{VO}_{2}$ max in patients with heart failure $(r=0.65 ; p=0.011)$ [76], pulmonary hypertension, $(r=0.70 ; p<0.001)$ [47], and idiopathic pulmonary fibrosis $(r=0.75 ; p<0.0001)$ [30]. Standard clinical measures which are routinely used to assess cardiopulmonary function such as oxygen saturation and forced vital capacity (FVC) are also strongly correlated with maximal $\mathrm{VO}_{2}[37,41,54]$.

Six-minute walk distances have also been statistically significantly correlated with other functional measures of cardiopulmonary reserves such as baseline cardiac output ( $r=0.48 ; p<0.05)$, total pulmonary resistance $(-0.49 ; p<0.05)$, oxygen pulse rate $(r=$ $0.57 ; p<0.001)$, and the ratio of minute ventilation to $\mathrm{CO}_{2}$ output (slope) $(r=-0.66 ; p<0.001)$ [47]. Although correlations between walk distance and mechanical efficiency of the skeletal musculature or markers of skeletal muscle oxidative capacity have not been directly measured, statistically significant associations between peak $\mathrm{O}_{2}$ consumption during exercise, which has been correlated with walk distance, and volume density of skeletal muscle mitochondria and surface density of mitochondrial cristae have been demonstrated in patients with chronic heart failure $(r=0.56 ; P<$ $0.001)$ [14].

Use of the 6-MWT as a tool for assessing the clinical course of disorders involving impairment of organ function has been validated in a prospective study of 898 patients with left ventricular dysfunction. A statistically significant 3-fold increase in mortality ( $p=$ 0.01 ) and 2-fold increase in hospitalization for heart failure ( $p=0.002$ ) over an 8-month follow-up period was observed among patients at the lowest performance level of the 6-MWT compared with those at the higher performance levels [4]. In multivariate analysis, a statistically significant relationship was demonstrated between baseline walk distance and mortality among patients with heart failure independent of the echocardiograpic, clinical, and neurohormonal parameters tested [47].

The 6-MWT has also been shown to be a highly reliable measurement of functional capacity in different patient populations. A reliability coefficient of 0.947 and coefficient of variation $(\mathrm{CV})$ of $10.4 \%$ were reported for repeat tests performed at 1-week intervals among patients with fibrotic idiopathic interstitial pneumonia [49]. A small training effect equivalent to about a $15 \%$ improvement in performance has been shown in patients who underwent testing on 2 consecutive days 1 week apart [17]. The reliability of the test is improved when subjects perform a practice test prior to the measurement to minimize the influence of familiarity with the test on the second measurement, as is currently recommended in standardized protocols [1, $8,17,22,50,54,67]$. A reduction in the $\mathrm{CV}$ from $8.2 \%$ to $4.2 \%$ was reported after the results of the first 2 of 6 repeat tests were eliminated from endurance assessments in patients with COPD [50]. The high intraclass correlations ( 0.96 to 0.99$)$ measured between a second and third walk test indicated that a single practice test was sufficient to improve reliability of the measure [7, 63].

The high variability $(30 \%)$ in walk distances measured in similar groups of patients across studies has been attributed to differences in the test protocols used in these studies [17]. Standardization of the type and the timing of encouragement given to patients during the test as well as reminders of the amount of time remaining can reduce the variability of the test results by as much as $30 \%$ [17,67]. Differences in age, gen- 
der, weight, and height in populations studied may also have contributed to the high variability reported for walk distances measured in similar groups of patients as these variables were shown to account for up to $40 \%$ of the variance in walk distances measured among healthy adults [18].

\section{Clinical significance of endurance testing}

Endurance tests are widely used in clinical settings to measure functional capacity in patients with pathology affecting the cardiovascular, pulmonary or musculoskeletal systems. Walk distances have been measured to assess severity and changes in severity of diseases such as chronic pulmonary obstructive disease [ 3 , $9,10,50,73$ ], pulmonary hypertension [47], idiopathic pulmonary fibrosis [39], idiopathic or fibrotic interstitial pneumonia $[18,42]$, congestive heart failure [38, $61,64,76]$, chronic heart failure [7,13,24,38,56,64,76], left ventricular dysfunction [4,70], stroke [49,53,56,57, $62,64,74]$, and mucopolysaccharidoses, including MPS VI $[2,6,26,28,29]$. Walk tests have also been used to evaluate treatment effects, predict short- and long-term clinical outcomes, confirm and quantify symptoms, and assess the functional limitations of patients with congestive and chronic heart failure [56,76]. Since functional capacity determines the ability to engage in activities encountered in daily life, endurance tests can be used as as a component of the assessment of quality of life [54]. Both functional capacity and quality of life are prognostic indicators of morbidity and mortality as well as of the psychosocial and economic impact of debilitating diseases $[1,4,9,13-15,25,26,38,39,42,46,47$, $49,54,56,61,64,70,75,76]$.

Diseases or congenital defects affecting cardiovascular, pulmonary, or musculoskeletal function reduce endurance capacity by compromising central hemodynamics, peripheral circulation, ventilatory efficiency, muscle strength, and joint range of motion [76]. In addition to early onset fatigue, patients with compromised cardiovascular function also frequently exhibit muscle weakness, palpitation, and chest pain during activity $[41,61,76]$. These symptoms can be attributed to an attenuation of the increase in stroke volume with increasing heart rate, reduced cardiac output [64], hemodynamic and metabolic abnormalities due to left ventricular dysfunction [70], inadequate tissue perfusion, decreased rate of aerobic metabolism, and inefficient regulation of core body temperature $[13,14,25$, $75,76]$.
Pulmonary diseases or structural defects that cause even small changes in the strength of the ventilator musculature $[41,42,46]$ or compliance of the lungs and thorax can exponentially increase the amount of effort required to breathe [41]. Under these conditions, a disproportionate amount of the energy produced will be consumed in breathing leaving only a small amount to support motor function and other metabolic activity important to performance [75,76]. Musculoskeletal system pathology such as mitochondrial and nonmetabolic myopathies can disrupt the energetics and mechanical efficiency of skeletal muscle [26]. Muscle atrophy and weakness, restricted joint range of motion, and skeletal deformities also make movement more difficult and painful, interfering with the ability to participate in and sustain activity.

\section{Endurance measures as prognostic indicators in MPS VI}

The 6-MWT, 12-MWT and 3-MSC are routinely used to assess the extent of disability and progression of disease in patients with MPS VI. Because MPS VI is a progressive disease with an underlying pathology that severely affects functioning of multiple organ systems, these tests are especially effective tools for evaluating responsiveness to treatment of this disease. As global assessment tools, these tests capture the full impact of multiple system deficits and because they are performed at submaximal intensity, they are sensitive indicators of endurance in MPS VI patients who are utilizing near maximal capacity to perform basic tasks $[12,16,21,51$, $60,66,68]$. Although standard clinical measurements correlate well with tests requiring maximal effort, they do not predict the extent of performance impairment in tests that require prolonged submaximal effort and thus are not as good prognostic indicators of clinical outcome as endurance measures [44].

Both cross-sectional and longitudinal studies have shown correlations between changes in 6-minute walk distances and changes in indicators of functional capacity and in disease progression in patients with MPS VI. A decrease in walk distance on the 6-MWT was linked to increased severity of symptoms experienced during the test including exertional dyspnea, tachycardia, tachypnea, and orthopenia [71]. Walk distances have been found to be consistently below average in patients with below normal values for pulmonary function based on FVC and below normal values for degree of joint restriction based on shoulder flexion and exten- 
sion tests. The results of longitudinal studies in MPS VI patients showed that increased walk distances generally paralleled decreases in urinary GAG excretion, indicating that improvements in endurance assessed by walk tests were reflective of favorable changes in this biological marker of disease severity [27,71]. The combined results of 3 clinical studies which evaluated endurance in a total of 56 MPS VI patients treated with enzyme replacement therapy (ERT) for up to 260 weeks showed sustained improvements from baseline in 6- and 12minute walk distances and in both the number and the rate of stairs climbed [27]. The increases in 12-minute walk distances were $246 \mathrm{~m}$ at Week $96(p<0.001)$ and $255 \mathrm{~m}$ at Week $144(p<0.001)$. These improvements paralleled the reductions in urinary GAG excretion observed over the same periods which ranged from 42$84 \%$ of baseline values. Increases in walk distances have also been shown to be consistent with improvements in results of pulmonary function and shoulder joint range of motion tests, supporting a general trend towards improvement in disease status with increased endurance capacity [71].

The correlations between changes in walk distances and changes in both the severity of symptoms and levels of urinary GAG support the clinical application of walk tests for evaluation of responsiveness to different treatment modalities including ERT in MPS VI patients [26, $28,36]$. The range of 6-minute walk distances that have been reported for this patient population is between $5 \mathrm{~m}$ and $270 \mathrm{~m}$ reflecting the large differences in severity of the disease and extent of organ involvement in these patients. Patients who walked the shorter distances at baseline had higher urinary GAG levels consistent with an expected low endurance capacity in patients with more severe disease [29]. Progressive improvements in walk distances with increasing treatment duration have also been documented. After 24 weeks of treatment, walk distances ranged from $200 \mathrm{~m}$ to $300 \mathrm{~m}$. The incremental increases in walk distances from baseline values after 24 weeks of treatment ranged from $43 \mathrm{~m}$ to $64 \mathrm{~m}$ and further increased to between $61 \mathrm{~m}$ and $75 \mathrm{~m}$ after 48 weeks $[26,28,29]$. The largest gains were observed among patients who were unable to walk 100 meters at baseline. As a percentage of baseline values, these changes amounted to an approximately $60 \%$ to $80 \%$ improvement in walk distance and were associated with an increase in urinary GAG excretion of $76 \%$ and an improvement of at least $50 \%$ in subjective assessments of joint pain and stiffness.

A relationship between walk distances measured by either the 6-MWT or 12-MWT and changes in clini- cal and biochemical measures of disease severity was confirmed from the results of a randomized, doubleblind, placebo-controlled study evaluating response to ERT over a 24-week period in 39 patients with MPS VI [26]. Relative to baseline values, the mean increase in walk distance measured at the 6-minute time point for patients in the treatment group was $53 \mathrm{~m}(95 \% \mathrm{CI}$ : $14 \mathrm{~m}, 90 \mathrm{~m}$ ) compared with no change in the placebo group ( $p=0.007)$. At the 12 -minute time point, mean distance walked was $92 \mathrm{~m} \pm 40 \mathrm{~m}(95 \% \mathrm{CI} ; 11 \mathrm{~m}$, $172 \mathrm{~m}$ ) farther for patients in the treatment group compared with patients in the placebo group $(p=0.025)$. In addition, walk distances continued to increase for patients in the treatment group over the next 24 weeks of open-label treatment, paralleling the decrease in urinary GAG observed.

The improvements in walk distances measured in MPS VI patients in these studies, although comparatively small, were highly clinically significant for this group of severely impaired patients. Because the extent of organ system involvement and the degree of functional deficits in different patient populations vary with disease pathology, the changes in walk distances observed in one patient population will not have the same clinical relevance when applied to another. For example, although increases of $70 \mathrm{~m}$ may not be clinically important for some patients, they can be indicative of a meaningful improvement in functional capacity and quality of life in severely compromised patients with diseases such as with COPD, who have baseline walk distances that are $80 \%$ of predicted values $[1,9$, 59]. The comparatively lower values of walk distances measured in MPS VI patients relative to patients with cardiovascular or pulmonary diseases may be explained by the multiple organ pathology and structural abnormalities characteristic of MPS VI and the young ages of the patients.

The majority of data on the use and interpretation of the 6-MWT reported in the literature has been obtained from adult patients with deficits in either cardiac function or pulmonary function. These patients are typically much older than patients with MPS VI and their diseases are not associated with structural malformations of the heart or lungs, nor do they involve skeletal abnormalities. Because MPS VI patients have an intrinsically lower functional capacity than other patient populations due to congenital defects, it would not be appropriate to evaluate changes in walk distances in these patients relative to other groups of patients who utilize a lower proportion of their maximal endurance capacity to perform routine tasks. Consequently, in patients 
with severely compromised organ system function such as in MPS VI, the changes in distance walked from baseline do not have to be large to have clinical significance. Furthermore, perceived improvement in walking ability by these as well as other groups of patients may be of greater clinical significance for interpreting the effectiveness of symptomatic treatments than large statistically significant increases. In a study of 112 patients with COPD, 6-minute walk distance was significantly correlated with these patients' subjective ratings of walking ability relative to their perception of other patients walking abilities $(r=0.59,95 \%$ confidence interval [CI]: 0.54 to 0.63 ) [59]. Changes of $54 \mathrm{~m}$ in 6-minute walk distance were associated with a noticeable improvement in subjective comparison ratings of walking ability in this study (95\% CI: 37 to $71 \mathrm{~m}$ ).

\section{Summary and conclusions}

Measurements of endurance capacity at submaximal intensity levels are considered to be more representative of the effort required to perform basic tasks in patients who require near maximal functional reserves to complete these tasks than standard physiological or clinical parameters. Walk tests have been shown to be valid and reliable tools for assessing functional status at submaximal intensity levels in different patient populations with compromised cardiopulmonary and musculoskeletal reserves, and are the most appropriate tests for assessment of functional deficits involving multiple organ systems. Walk tests are simple and inexpensive tests to administer and safer than most other endurance tests for patients with severely compromised cardiopulmonary and musculoskeletal function because the level of exertion required is self- and symptom-limited to the level of effort achievable by each patient [15, 47]. Limitations of the walk test include the impact of learning effects and motivational factors such as encouragement and fear on test scores. These limitations can be addressed by practice tests and by standardization of the testing protocol to account for the influence of motivational factors. Walk tests are feasible to perform in children and older patients with multiple organ system impairments and structural abnormalities that limit their ability to perform more complex endurance tests and thus are appropriate for evaluating treatment responses in children with MPS VI.

Six minute walk distances have been correlated with standard measures of cardiac and pulmonary functional reserves that are widely accepted predictors of changes in physiological markers of improvement in endurance capacity such as $\mathrm{VO}_{2}$ max and oxygen saturation. Sixminute walk distances have been shown to be strong and independent prognostic indicators for short and long-term morbidity and mortality in patients with cardiopulmonary diseases. In evaluating responsiveness to treatment, changes in 6-minute walk distances do not need to be large to be clinically relevant since small improvements can reflect meaningful changes in the ability to perform routine tasks of daily living. The greater independence that even small changes represent improves the quality of life, which is an important treatment goal for patients with chronic degenerative diseases.

\section{Acknowledgements}

Arline McDonald and MediResource Inc. received funding for writing and editorial assistance from BioMarin Pharmaceutical Inc., Novato, CA. Dr. Helen Nicely of BioMarin contributed to the editing of this document. Dr. Steiner has in the past provided consulting support to BioMarin Pharmaceutical Inc., Novato, CA. Sean Turbeville is an employee and stockholder of BioMarin Pharmaceutical Inc., Novato, CA.

\section{References}

[1] ATS statement: guidelines for the six-minute walk test, Am J Respir Crit Care Med 166(1) (2002), 111-117.

[2] I.M. Balfour-Lynn, S.A. Prasad et al., A step in the right direction: assessing exercise tolerance in cystic fibrosis, Pediatr Pulmonol 25(4) (1998), 278-284.

[3] M.L. Bernstein, J.A. Despars et al., Reanalysis of the 12minute walk in patients with chronic obstructive pulmonary disease, Chest 105(1) (1994), 163-167.

[4] V. Bittner, D.H. Weiner et al., Prediction of mortality and morbidity with a 6-minute walk test in patients with left ventricular dysfunction, SOLVD Investigators, JAMA 270(14) (1993), 1702-1707.

[5] J.K. Bredenkamp, M.E. Smith et al., Otolaryngologic manifestations of the mucopolysaccharidoses, Ann Otol Rhinol Laryngol 101(6) (1992), 472-478.

[6] R.J. Butland, J. Pang et al., Two-, six-, and 12-minute walking tests in respiratory disease, $\mathrm{Br}$ Med J (Clin Res Ed) 284(6329) (1982), 1607-1608.

[7] L.P. Cahalin, M.A. Mathier et al., The six-minute walk test predicts peak oxygen uptake and survival in patients with advanced heart failure, Chest 110(2) (1996), 325-332.

[8] L. Cahalin, P. Pappagianopoulos et al., The relationship of the 6-min walk test to maximal oxygen consumption in transplant candidates with end-stage lung disease, Chest 108(2) (1995), 452-459. 
[9] R. Carter, D.B. Holiday et al., Tiep, 6-minute walk work for assessment of functional capacity in patients with COPD, Chest 123(5) (2003), 1408-1415.

[10] M.L. Chuang, I.F. Lin et al., The body weight-walking distance product as related to lung function, anaerobic threshold and peak VO2 in COPD patients, Respir Med 95(7) (2001), 618626.

[11] K.H. Cooper, A means of assessing maximal oxygen intake, JAMA 203 (1968), 201-204.

[12] J.H. Dangel, Cardiovascular changes in children with mucopolysaccharide storage diseases and related disordersclinical and echocardiographic findings in 64 patients, Eur $J$ Pediatr 157(7) (1998), 534-538.

[13] S.W. Davies and D.P. Lipkin, Exercise physiology and the role of the periphery in cardiac failure, Curr Opin Cardiol 7(3) (1992), 389-396.

[14] H. Drexler, U. Riede et al., Alterations of skeletal muscle in chronic heart failure, Circulation 85(5) (1992), 1751-1759.

[15] T. Eaton, P. Young et al., Six-minute walk, maximal exercise tests: reproducibility in fibrotic interstitial pneumonia, Am J Respir Crit Care Med 171(10) (2005), 1150-1157.

[16] J. Engle, H.J. Safi et al., Mucopolysaccharidosis presenting as pediatric multiple aortic aneurysm: first reported case, $J$ Vasc Surg 26(4) (1997), 704-710.

[17] P.L. Enright, The six-minute walk test, Respir Care 48(8) (2003), 783-785

[18] P.L. Enright and D.L. Sherrill, Reference equations for the six-minute walk in healthy adults, Am J Respir Crit Care Med 158(5 Pt 1) (1998), 1384-1387.

[19] B. Fernhall and V.B. Unnithan, Physical activity, metabolic issues, and assessment, Phys Med Rehabil Clin N Am 13(4) (2002), 925-947.

[20] R. Giugliani, P. Harmatz and J.E. Wraith, Management Guidelines for Mucopolysaccharidosis VI, Pediatrics 120(2) (2007), 405-418.

[21] D.M. Gross, J.C. Williams et al., Echocardiographic abnormalities in the mucopolysaccharide storage diseases, Am J Cardiol 61(1) (1988), 170-176.

[22] G.V. Guimaraes, G. Bellotti et al., Can the cardiopulmonary 6-minute walk test reproduce the usual activities of patients with heart failure? Arq Bras Cardiol 78(6) (2002), 553-560.

[23] M. Gulati and P.E. McBride, Functional capacity and cardiovascular assessment: submaximal exercise testing and hidden candidates for pharmacologic stress, Am J Cardiol 96(8A) (2005), 11J-19J.

[24] G.H. Guyatt, M.J. Sullivan et al., The 6-minute walk: a new measure of exercise capacity in patients with chronic heart failure, Can Med Assoc J 132(8) (1985), 919-923.

[25] R. Hambrecht, J. Niebauer et al., Physical training in patients with stable chronic heart failure: effects on cardiorespiratory fitness and ultrastructural abnormalities of leg muscles, $\mathrm{J} \mathrm{Am}$ Coll Cardiol 25(6) (1995), 1239-1249.

[26] P. Harmatz, R. Giugliani et al., Enzyme replacement therapy for mucopolysaccharidosis VI: a phase 3, randomized, doubleblind, placebo-controlled, multinational study of recombinant human $\mathrm{N}$-acetylgalactosamine 4-sulfatase (recombinant human arylsulfatase B or rhASB) and follow-on, open-label extension study, J Pediatr 148(4) (2006), 533-539.

[27] P. Hartmatz, R. Giugliani et al., Long-term follow-up of endurance and safety outcomes during enzyme replacement therapy for mucopolysaccharidosis VI: Final results of three clinical studies of recombinant human N-acetylgalactosamine-4sulfatase, Mol Genet Metab 94(4) (2008), 469-475.
[28] P. Harmatz, D. Ketteridge et al., Direct comparison of measures of endurance, mobility, and joint function during enzyme-replacement therapy of mucopolysaccharidosis VI (Maroteaux-Lamy syndrome): results after 48 weeks in a phase 2 open-label clinical study of recombinant human $\mathrm{N}$-acetylgalactosamine 4-sulfatase, Pediatrics 115(6) (2005), e681-e689.

[29] P. Harmatz, C.B. Whitley et al., Enzyme replacement therapy in mucopolysaccharidosis VI (Maroteaux-Lamy syndrome), $J$ Pediatr 144(5) (2004), 574-580.

[30] E. Herskhovitz, E. Young et al., Bone marrow transplantation for Maroteaux-Lamy syndrome (MPS VI): long-term followup, J Inherit Metab Dis 22(1) (1999), 50-62.

[31] D.L. Huggett, D.M. Connelly et al., Maximal aerobic capacity testing of older adults: a critical review, J Gerontol A Biol Sci Med Sci 60(1) (2005), 57-66.

[32] M.J. Joyner and E.F. Coyle, Endurance Exercise Performance: The Physiology of Champions, J Physiol (2007), September 27.

[33] C. Kearon, G.R. Viviani et al., Factors influencing work capacity in adolescent idiopathic thoracic scoliosis, Am Rev Respir Dis 148(2) (1993), 295-303.

[34] J.O. Kelly, S.L. Kilbreath et al., Cardiorespiratory fitness and walking ability in subacute stroke patients, Arch Phys Med Rehabil 84(12) (2003), 1780-1785.

[35] S. King, J. Wessel et al., Validity and reliability of the 6 minute walk in persons with fibromyalgia, J Rheumatol 26(10) (1999), 2233-2237.

[36] W. Krivit, M.E. Pierpont et al., Bone-marrow transplantation in the Maroteaux-Lamy syndrome (mucopolysaccharidosis type VI). Biochemical and clinical status 24 months after transplantation, N Engl J Med 311(25) (1984), 1606-1611.

[37] V.N. Lama, K.R. Flaherty et al., Prognostic value of desaturation during a 6-minute walk test in idiopathic interstitial pneumonia, Am J Respir Crit Care Med 168(9) (2003), 1084-1090.

[38] A.I. Larsen, T. Aarsland et al., Assessing the effect of exercise training in men with heart failure; comparison of maximal, submaximal and endurance exercise protocols, Eur Heart $J$ 22(8) (2001), 684-692.

[39] D.J. Lederer, S.M. Arcasoy et al., Six-minute-walk distance predicts waiting list survival in idiopathic pulmonary fibrosis, Am J Respir Crit Care Med 174(6) (2006), 659-664.

[40] V. Margonato, F. Fronte et al., Effects of short term cast wearing on respiratory and cardiac responses to submaximal and maximal exercise in adolescents with idiopathic scoliosis, $E u$ ra Medicophys 41(2) (2005), 135-140.

[41] W.D. McArdle, F.I. Katch et al., The Physiologic Support Systems, in: Essentials of Exercise Physiology, Third ed., Lippincott Williams \& Wilkins, Philadelphia, 2006, pp. 290431.

[42] W.D. McArdle, F.I. Katch et al., Energy Transfer, in: Essentials of Exercise Physiology, Lippincott Williams \& Wilkins, Philadelphia, 2007, pp. 166-288.

[43] C.R. McGavin, M. Artvinli et al., Dyspnoea, disability, and distance walked: comparison of estimates of exercise performance in respiratory disease, $\mathrm{Br}$ Med J 2(6132) (1978), 241-243.

[44] C.R. McGavin, S.P. Gupta et al., Twelve-minute walking test for assessing disability in chronic bronchitis, $\mathrm{Br}$ Med J1(6013) (1976), 822-823.

[45] A. McManus and M. Leung, Maximising the clinical use of exercise gaseous exchange testing in children with repaired cyanotic congenital heart defects: the development of an appropriate test strategy, Sports Med 29(4) (2000), 229-244. 
[46] M. Metra, S. Nodari et al., Maximal and submaximal exercise testing in heart failure, J Cardiovasc Pharmacol 32(Suppl 1) (1998), S36-S45.

[47] S. Miyamoto, N. Nagaya et al., Clinical correlates and prognostic significance of six-minute walk test in patients with primary pulmonary hypertension. Comparison with cardiopulmonary exercise testing, Am J Respir Crit Care Med 161 (2000), 487-492.

[48] R. Mocellin, Exercise testing in children with congenital heart disease, Pediatrician 13(1) (1986), 18-25.

[49] P.S. Montgomery and A.W. Gardner, The clinical utility of a six-minute walk test in peripheral arterial occlusive disease patients, J Am Geriatr Soc 46(6) (1998), 706-711.

[50] I.P. Mungall and R. Hainsworth, Assessment of respiratory function in patients with chronic obstructive airways disease, Thorax 34(2) (1979), 254-258.

[51] J. Nelson, M.D. Shields et al., Cardiovascular studies in the mucopolysaccharidoses, J Med Genet 27(2) (1990), 94-100.

[52] E. Neufeld and J. Muenzer, The mucopolysaccharidoses, in: The Metabolic Basis of Inherited Disease, McGraw-Hill, 1995, pp. 2465-2493.

[53] H. Nielens, V. Boisset et al., Fitness and perceived exertion in patients with fibromyalgia syndrome, Clin J Pain 16(3) (2000), 209-213.

[54] V. Noonan and E. Dean, Submaximal exercise testing: clinical application and interpretation, Phys Ther 80(8) (2000), 782807.

[55] E. Paap, N.J. van der et al., Physiologic response of the sixminute walk test in children with juvenile idiopathic arthritis, Arthritis Rheum 53(3) (2005), 351-356.

[56] A. Passantino, R. Lagioia et al., Short-term change in distance walked in $6 \mathrm{~min}$ is an indicator of outcome in patients with chronic heart failure in clinical practice, $J$ Am Coll Cardiol 48(1) (2006), 99-105.

[57] P.S. Pohl, P.W. Duncan et al., Influence of stroke-related impairments on performance in 6-minute walk test, $J$ Rehabil Res Dev 39(4) (2002), 439-444.

[58] E.J. Protas, T.G. Mayer et al., Relevance of aerobic capacity measurements in the treatment of chronic work-related spinal disorders, Spine 29(19) (2004), 2158-2166.

[59] D.A. Redelmeier, A.M. Bayoumi et al., Interpreting small differences in functional status: the Six Minute Walk test in chronic lung disease patients, Am J Respir Crit Care Med 155(4) (1997), 1278-1282.

[60] D. Rigante and G. Segni, Cardiac structural involvement in mucopolysaccharidoses, Cardiology 98(1-2) (2002), 18-20.

[61] P. Rossi, Physical training in patients with congestive heart failure, Chest 101 (1992), 350S-353S.
[62] C. Rostagno, G. Olivo et al., Prognostic value of 6-minute walk corridor test in patients with mild to moderate heart failure: comparison with other methods of functional evaluation, Eur J Heart Fail 5(3) (2003), 247-252.

[63] A. Roth-Isigkeit, U. Thyen et al., Pain among children and adolescents: restrictions in daily living and triggering factors, Pediatrics 115(2) (2005), e152-e162.

[64] G. Roul, P. Germain et al., Does the 6-minute walk test predict the prognosis in patients with NYHA class II or III chronic heart failure? Am Heart J 136(3) (1998), 449-457.

[65] S. Savci, Inal-Inc et al., Six-minute walk distance as a measure of functional exercise capacity in multiple sclerosis, Disabil Rehabil 27(22) (2005), 1365-1371.

[66] R.M. Schieken, R.E. Kerber et al., Cardiac manifestations of the mucopolysaccharidoses, Circulation 52(4) (1975), 700705 .

[67] J.C. Sclafani, The Six-Minute Walk Test, AARC Times (pamphlet) (2000), 68-71.

[68] G.L. Semenza and R.E. Pyeritz, Respiratory complications of mucopolysaccharide storage disorders, Medicine (Baltimore) 67(4) (1988), 209-219.

[69] D. Singh-Grewal, V. Wright et al., Feldman BM. Pilot study of fitness training and exercise testing in polyarticular childhood arthritis, Arthritis Rheum 55(3) (2006), 364-372.

[70] M.J. Sullivan, M.B. Higginbotham et al., Exercise training in patients with severe left ventricular dysfunction. Hemodynamic and metabolic effects, Circulation 78(3) (1988), 506-515.

[71] S.J. Swiedler, M. Beck et al., Threshold effect of urinary glycosaminoglycans and the walk test as indicators of disease progression in a survey of subjects with Mucopolysaccharidosis VI (Maroteaux-Lamy syndrome), Am J Med Genet A 134(2) (2005), 144-150.

[72] T. Taivassalo, H. Reddy et al., Muscle responses to exercise in health and disease, Neurol Clin 18(1) (2000), 15-34.

[73] H.A. van Helvoort, Y.F. Heijdra et al., Six-minute walkinginduced systemic inflammation and oxidative stress in musclewasted COPD patients, Chest 131(2) (2007), 439-445.

[74] K.E. Wallman, A.R. Morton et al., Physiological responses during a submaximal cycle test in chronic fatigue syndrome, Med Sci Sports Exerc 36(10) (2004), 1682-1688.

[75] J.R. Wilson and D.M. Mancini, Factors contributing to the exercise limitation of heart failure, J Am Coll Cardiol 22(4 Suppl A) (1993), 93A-98A.

[76] D.J. Wright and L.B. Tan, The role of exercise testing in the evaluation and management of heart failure, Postgrad Med J 75(886) (1999), 453-458.

[77] J.W. Ziegler, Exercise-limiting symptoms in children, Med Health R I 90(8) (2007), 240-248. 